\title{
Coverage Methods for Early Groundwater Contamination Detection
}

\author{
Luís Miguel Nunes ${ }^{1}$ \\ Maria da Conceição Cunha
}

Luís Ribeiro

\author{
Faculty of Sciences and Technology, University of \\ Algarve, Faro, Portugal. e-mail: lnunes@ualg.pt \\ University of Coimbra, Coimbra, Portugal. e-mail: \\ mccunha@dec.uc.pt \\ Instituto Superior Técnico, Lisbon Technical University, \\ Lisboa, Portugal. e-mail: luis.ribeiro@ist.utl.pt
}

\begin{abstract}
A method based on space-filling coverage designs to optimize groundwater monitoring networks for plume detection and quantification is proposed. Space-filling objective functions are then compared with more classical functions. The method was applied to a hypothetical case-study with 160 candidate locations, resulting in final optimal design monitoring networks with 40 locations. Results show that the method is superior to those based strictly on the probability of contamination detection for quantifying maximum and mean values. In the light of these results fractal properties of space-filling coverage methods and of simulated annealing are also discussed.
\end{abstract}

11 Keywords: space-filling, groundwater, monitoring

A vast number of technical documentation has been published on the design and operation of groundwater monitoring networks, namely in respect to where monitoring points should be located and which sampling strategies to adopt. Four techniques are used to tackle these questions. The first is based on geostatistical methods (Rouhani 1985), and the second on simulation methods, also from the late 1980s (Massmann and Freeze 1987). The third category of techniques to appear involved transfer functions (Andricevic 1990). However, the most widely used approaches are optimization methods, which were first introduced in the early eighties (Olea 1999), being latter developed by many other authors, with the later models gradually incorporating objective functions with cost parameters, such as, installation, operation, maintenance and environmental costs (Reed et al. 2000), or the minimization of the number of wells (Meyer et al. 1994). A detection monitoring network is optimal if its capacity to detect early contamination is maximal (Meyer et al. 1994) and the concentration levels are very low. A compliance monitoring network is one that is able to give the best representation of the effective concentrations best represents the spatial distribution of the variable (Cunha and Nunes 2011). Some simplifications to least-cost objective functions have been proposed, in particular by using proxies, like minimizing the error of kriged concentration values, or the variance of the error of the kriged concentration error (Nunes et al. 2004b). We concentrate here on another proxy method, the space-filling methods, for which this is the first application in 34 groundwater monitoring. Space-filling functions have already been used in the design of

${ }^{1}$ Corresponding author 
air quality monitoring network design by Morris and Mitchell (1995) and by Royle and Nychka (1998). As the decision variables (location of the monitoring site) are combinatorial, the models contain discrete variables and so the classic linear, nonlinear and integer linear programming methods are unsuitable. Lee and Ellis (1996) concluded that simulated annealing and tabu search perform best for groundwater monitoring network design.

One of the hardest tasks in groundwater contamination evaluation is to characterize contamination plumes, because a large number of sampling sites is generally required to obtain good estimates (accurate and exact) of the contaminated area and concentration values. The practical difficulty with these estimates is directly related to the uncertainty about many of the flow and transport parameters. At the top of the list are the medium's hydraulic conductivity and dispersivity. Hydraulic conductivity affects groundwater flow velocities at all scales, which, as a result, will also condition contaminant dispersion (calculated as the product of flow velocity and dispersivity). These uncertainties about parameters (state-variables) should be incorporated into the modeling to best reflect uncertain decisions about parameter values. The uncertainty is well handled by statistical approaches, where a state-variable spatial distribution is considered a random function, the value at a given location is a random variable, and the sampled values are a possible outcome of the random variable. There are two main approaches to groundwater modeling using random fields of medium parameters. One is the expansion of the uncertain parameters in terms of a series. The other is stochastic simulations based on Monte Carlo methods, of which the most common are Latin hypercube sampling, sequential Gaussian simulations, turning bands method, and LU decomposition.

In the present paper a method for optimizing monitoring networks for detecting and estimating the shape of the plumes is presented. The method combines space-filling methods and Monte-Carlo sequential simulations. Two objective functions are compared: i) includes both the space-filling criterion and the relative number of contamination detections criterion; ii) includes only the relative number of contamination detections criterion. The latter is similar to the objective functions proposed by other authors as the "probability of contamination detection" criterion (James and Gorelick 1994). A specific computed code in FORTRAN was developed by the team to test monitoring optimization problems. Some very preliminary results were presented in Nunes et al. (2005).

\section{Materials and methods}

The method requires the simulation of $L$ alterative concentration fields by modeling mass transport in an equal number of hydraulic conductivity random fields, in which $\Omega$ locations are placed (Step 1) - candidate set $\mathbf{C}$; in Step 2 a set of $\omega$ locations is chosen from $\mathbf{C}$, generating design set $\mathbf{D}$ (solution generation); in Step 3 the number of detections and the space-filling criterion are computed using the design set, and the objective function (OF) is computed. Convergence of the objective function to the optimal value is

77 functrolled by the simulated annealing algorithm, responsible for controlling the entire 79 process starting in Step 2: the process of solution generation and OF calculation is 
cyclical until the criteria for stopping the algorithm are attained and the optimal solution

81 presented (see Figure 1).

\section{Figure 1 Method}

The candidate sets $\mathbf{C}$ are obtained by making mass transport simulations of contaminant dispersion in groundwater and considering the sampling locations as the values in the model nodes. Uncertainty is introduced by generating several conditional simulations of the hydraulic conductivity field using sequential Gaussian simulation. The method is conveniently offered by the GSLIB geostatistical toolbox (Deutsch and Journel 1992). Applying the deterministic groundwater flow to these random fields will result in hydraulic potential fields and velocity fields that are also random functions. Solving flow and transport equations with the proper initial and boundary conditions simulates the transport of a chemical species If enough stochastic simulations are computed and modeled, then it will be possible to compute, at each model cell, the density function of contaminant concentration, and also the probability that a given threshold is surpassed. Consider the concentration a chemical species study, $C\left(x_{\mathrm{i}, \mathrm{j}}\right)$, at a location $x_{\mathrm{i}, \mathrm{j}}$, and a reference value (e.g., legal limit, or analytical detection limit), $C_{\text {ref, }}$ then, the relative number of detections is determined by

$$
\begin{aligned}
& a_{m}\left(x_{i, j}\right)=\left\{\begin{array}{ll}
1 & \text { if } C_{m}\left(x_{i, j}\right) \geq C_{r e f} \\
0 & \text { otherwise }
\end{array} \quad m=1, \ldots, L\right. \\
& r\left(x_{i, j}\right)=\frac{1}{L} \sum_{m=1}^{L} a_{m}\left(x_{i, j}\right)
\end{aligned}
$$

100 The use of $r\left(x_{\mathrm{i}, \mathrm{j}}\right)$ reflects the empirical need to include in the monitoring network those 101 stations that detected contamination more often, i.e., with higher detection capacity. A 102 good space-filling design is one with monitoring locations scattered throughout the 103 domain with minimal unsampled areas (Fang et al. 2000). Space-filling methods use a 104 criterion based on a metric that makes it possible to evaluate the goodness of a space 105 covering design. The most common criteria are based on the average of distances 106 between candidate locations and the locations already included in the design sub-set 107 (equation (4)). One possible metric is given by $d_{p}(x, \boldsymbol{D})$.

$$
\Pi_{p, q}(\mathbf{D})=\left(\sum_{u \in \mathbf{C}} d_{p}(x, \mathbf{D})^{q}\right)^{1 / q}, \text { with } d_{p}(x, \mathbf{D})=\left(\sum_{u \in \mathbf{D}}\|x-u\|\right)^{1 / p}
$$

108 The exponent $q$ is $>0$ and is $p<0 . d_{p}(x, \mathbf{D}) \rightarrow 0$ as the location $x$ converges to a member of 109 D. The coverage design is the subset of $\omega$ elements in $\mathbf{D}$ from the $\Omega$ elements in $\mathbf{C}, \mathbf{D} \subset$ $110 \mathbf{C}$, that minimize the criterion $\Pi_{p, q}(\mathbf{D})$. The algorithm implemented in our computer code 111 is a simulated annealing equivalent of the exchange (or swap) algorithm as proposed by 112 Johnson et al. (1990) considering no restriction on neighborhood search. The two 113 objective functions studied here are 


$$
\begin{aligned}
& \text { (M1) } \min \frac{\Pi_{p, q}(\mathbf{D})}{\sum_{i} \sum_{j} r\left(x_{i, j}\right)} \\
& \text { (M2) } \max \sum_{i} \sum_{j} r\left(x_{i, j}\right)
\end{aligned}
$$

114 M1 was constructed so as to combine the best characteristics of the "probability of 115 contamination detection" criterion, and the space-filling criterion. With the first, 116 maximization of contamination detection is sought; with the second criterion, 117 maximization of space coverage is intended. Hence, the resulting monitoring networks 118 should allow good estimates of contaminated areas, and good contamination detection 119 capacity. M2 has been used by many other authors in other methodological approaches, 120 and is used here for benchmarking the first model. The problem proposed here was 121 solved using a simulated annealing (SA) heuristic optimization algorithm, executed in 122 Fortran 90. The implementation followed the description presented in Nunes et al. 123 (2004a).

The proposed objective function models were tested in a hypothetical case-study, consisting of a continuous source of a conservative chemical species, located in a very small area, which contaminates a porous unconfined aquifer. This example illustrates, e.g., the leakage from storage tanks, or from landfills, of a chemical species that does not adsorb to the soil matrix nor is it affected by degradation, or does so in a very limited fraction. The problem usually faced in these cases is where to locate the monitoring piezometers so that they have the greatest probability of detecting the contamination, also allowing the best estimation of the plume concentration geometry (estimation of the affected area and volume). The problem that is solved here is one of detection and evaluation of spread monitoring after the early detection of a rupture in an underground containment structure, or a spill on the soil surface. The period between leakage and setting of the monitoring network for early assessment of contamination is 30 days, corresponding to the amount of time elapsing between first detection, decision to undertake the monitoring, contracting the service, and setting up the monitoring network. The modeling domain is, in this simplified example, a rectangle of $400 \mathrm{~m} \times 150 \mathrm{~m}$, discretized into a $10 \mathrm{~m}$ squared mesh, in $2 \mathrm{D}$ conditions. Upper and lower limits of the porous medium are horizontal and the depth of the aquifer is $20 \mathrm{~m}$. Flow boundary conditions of Dirichlet type with a head value at $27 \mathrm{~m}$ on the West boundary and at $24 \mathrm{~m}$ on the East boundary. No pumping and no recharge are considered. Simulation time is 30 days. Given the fact that modeling conditions do not change during the modeling period, steady-state conditions are used. Hydraulic conductivity is a heterogeneous stochastic field with mean hydraulic conductivity, $K$, of $2.12 \times 10^{-2} \mathrm{~m} / \mathrm{s}$, and variance of hydraulic conductivity of $2.5 \times 10^{-1} \mathrm{~m}^{2} / \mathrm{s}^{2}$, modeled with an isotropic spherical variogram (nugget $=$ 1480.01 ; sill $=0.24$; range $=80 \mathrm{~m}$ ). Effective porosity is considered constant and equal to 0.1 149 throughout the domain, whatever the value of $K$. The longitudinal dispersivity coefficient 150 is $4.5 \mathrm{~m}$, with anisotropy ratio, $\alpha_{y} / \alpha_{x}$, of 0.25 . The amount of the chemical species 151 entering at the top the water table is $400 \mathrm{~g} / \mathrm{m}^{2} . \mathrm{d}$, modeled as a contaminated recharge over an area of $100 \mathrm{~m}^{2}$. No retardation or degradation is considered (given the short time length considered, the latter assumption is valid even for the most readily biodegradable species). The molecular diffusion coefficient is considered irrelevant, given the groundwater flow velocity. At the onset of the simulation concentrations of the chemical 
species inside the domain are zero. Groundwater potentials (equation (1)) in the domain are simulated with MODFLOW (McDonald and Harbaugh 1988). Concentrations are simulated with the MT3D code (Zheng 1990), which solves equation (2) for conservative chemical species. Calculation of the value of $r\left(x_{\mathrm{i}, \mathrm{j}}\right)$ required carrying out 100 stochastic sequential Gaussian simulations and an equal number of flow and mass transport simulations. The reference value, $C_{\text {ref }}$, is $50 \mathrm{mg} / \mathrm{l}$. It is assumed that the candidate set of locations is known, with dimension $\Omega$, and that a design sub-set, with dimension $\omega$, is sought. The design problem is to find the optimal set of $\omega$ locations, $\mathbf{D}==\left(x_{i}: i=1,2, \ldots\right.$, $\omega)$, from a candidate set with $\Omega$ locations, $\mathbf{C}=\left(x_{j}: j=1,2, \ldots, \Omega\right)$. The candidate set has $\Omega=160$ locations, of which only $\omega=40$ locations are allowed in the optimal design monitoring network. The dimension of the solution space is given by the well-known equation $\Psi=\Omega ! /[(\Omega-\omega] ! \omega !]=8.6 \times 10^{37}$.

\section{Results and Discussion}

The objective function model M1 was studied for four different combinations of the coefficients $p$ and $q: n(p, q)=((-1,3),(-1,4),(-3,2),(-3,4))$. The choice of the coefficients is arbitrary. For instance, Royle and Nychka (1998) used $p=-5$ and $q=-1$, as a compromise between designs that are close to the minimax solution. In our case the combination $(-5,-$ 1) was found to be too instable to compute, giving no meaningful results in any of the 10 runs. It will be clear that for some combinations of $p$ and $q$ the convergence is much more difficult or even impossible in a practical amount of time. The results from the tested objective functions were compared with OF M2 to evaluate the impact of including the space-filling component in the objective function. If the problem had had to be solved exhaustively by testing all the possible combinations of locations it would require, with the $2 \mathrm{GHz}$ Pentium PC used in the calculus, more than $6.9 \times 10^{29}$ years. The solutions presented here, which are the best from 10 runs with different initial solutions, took nearly 7 hours to calculate each. When the algorithm converges, SA has been found to be an efficient optimization method. The disadvantage is that it is impossible to know if the good quality solution obtained by the algorithm is the global optimum (the minimum of the minima), because optimality is only guaranteed in an almost infinite number of iterations (almost infinite time). The advantages far outweigh the disadvantages, though. Figure 2 shows the convergence curves for two $(p, q)$ combinations. A large jump from high objective function values at high temperatures to very low values is evident for ($1,3)$. Since simulated annealing is a method based on physics annealing processes, when a material is cooled slowly into its crystallized form it is possible that it may show some characteristics also common in physics, like supersolid transition: a very fast drop in some characteristic of the material when cooled to below some critical temperature (Andreev and Lifshitz 1969). This may also be related to fractal properties of spacefilling networks, for which there seems to be a constant factor relating the dimension of the network (distance between locations or number of branches in a network) to its coverage area. What may be happening here is that the critical temperature is related in some way to specific spatial organizations that best approximate the fractal nature governing natural networks. This may become an important indication as to which solutions may be the best candidates to constitute a smaller solution space, if one can devise a method to identify a set of solutions with the correct fractal nature (e.g., similar fractal dimension). The behavior of simulated annealing depends crucially on the energy 
landscape associated with the optimization problem: the landscape must have special properties if annealing is to be efficient (fractalness) (Sorkin 1991). Figure 2 shows that for $(-2,3)$ the fractalness may not have been found, indicating that either a different transition rule could have been used to improve further the solution (e.g., 2 or 3-opt), and/or a different combination of this with a slower temperature decrease, but at the expense of longer running times. These results do not explore all possible combinations of M1 objective functions (nor is that possible), but they do show that it may be advisable to test some different objective functions before choosing a solution. For comparison purposes both the random field $r\left(x_{\mathrm{i}, \mathrm{j}}\right)$ obtained with the candidate sets $\mathbf{C}, r_{C}\left(x_{\mathrm{i}, \mathrm{j}}\right)$ and those obtained with the optimal $\mathbf{D}$ sets, $r_{\boldsymbol{D}}\left(x_{\mathrm{i}, \mathrm{j}}\right)$, were kriged in an area equal to the modeling domain, in a grid with $15 \times 39=585$ nodes, using the same variogram model as before. The kriged fields were then compared with the following statistics: i) mean; ii) maximum; iii) minimum; iv) mean estimation error; v) relative mean error $\left[\left(r_{D}\left(x_{\mathrm{i}, \mathrm{j}}\right)\right.\right.$ $\left.\left.r_{C}\left(x_{\mathrm{i}, \mathrm{j}}\right)\right) / 585\right]$; vi) mean estimation variance. When comparing the two solutions that converged, $(p, q)=(-1,3)$ or $(-2,3)$, with the other two that did not (not shown in the figure), it is clear that in terms of the quality of the reproduction of the spatial field, the former outperform the latter. This is indicated by a mean estimated $r_{D}\left(x_{\mathrm{i}, \mathrm{j}}\right)$ value closer to that of the candidate network, $r_{C}\left(x_{\mathrm{i}, \mathrm{j}}\right)$. It is interesting to see that the statistics (Table 1) for $(p, q)=(-3,4)$ are all at a very good level, with the exception of the mean estimated value, which is the worst of the four, and so the solution would have been a good one in a strict variance-reduction approach.

Table 1 Comparison of results for objective functions M1 and M2 (best of the ten runs)

Results showed that if the intuitive approach of including stations in the design is exclusively based on the highest relative number of detections (M2), the resulting network will tend to be too concentrated in the center of the plume (Figure 3c). Also in this case the quality of the estimated spatial field (of $r\left(x_{\mathrm{i}, \mathrm{j}}\right)$ (and therefore of the concentrations) is very poor, as shown by the indicator statistics in Table 1. This objective function has the worst results of the five.

Figure 2 Simulated annealing convergence curves (best of the ten runs)

If the objective function M1 is used, the network is much more evenly distributed in space, covering not only the center of the plume, but also areas where contamination levels are very low (see Figure 3). This is usually a much more realistic objective when dealing with the detection of contamination events from point or areal sources. Other methods have been proposed along these lines using geostatistics and exploration costs by (Cunha and Nunes 2011) with similar results. The advantage of the spacefilling/relative number of detections method over the variance-reduction method lies in its speed, because geostatistical simulations are made before the optimization, no kriging is needed during optimization, and because it is not constrained by ergodicity assumptions (not always verified in contaminated areas due to three-dimensional concentration trends). 
Figure 3 a) Relative number of detections, $r_{C}\left(x_{i, j}\right)$; b) M1; c) M2

The advantages over other methods is the simplicity and speed of its implementation, as well as its intuitively more reality-based approach, making it easier to convey to decision makers. It describes a method for optimizing monitoring networks for the detection and estimation of the shape of the plumes. The intuitive approach of including stations in the design exclusively based on the highest relative number of detections resulted in a monitoring network that is too concentrated in the center of the plume. If the objective function M1 is used, the network was revealed to be much more evenly distributed in space, covering not only the center of the plume, but also areas where contamination levels are very low This is a much more realistic result when dealing with the detection of contamination events from point or areal sources.

\section{References}

Andreev AF, Lifshitz IM (1969) Quantum theory of defects in crystals. Sov Phys JETPUSSR 29: 1107-1113

Andricevic R (1990) Cost-effective network design for groundwater-flow monitoring. Stoch Hydrol Hydraul 4(1): 27-41

Cunha MC, Nunes LM (2011) Groundwater systems: characterization, management and monitoring. WIT Press, Southampton, UK

Deutsch, CV, AG Journel (1992) GSLIB - Geostatistical software library and user's guide. Oxford University Press, New York, USA

Fang, KT, Lin DKJ, Winker P, Zhang Y (2000) Uniform Design: Theory and Application. Technometrics 42(3): 237-248

Johnson ME, Moore LM, Ylvisaker D (1990) Minimax and maximin distance designs. J Stat Plan Infer 26: 131-148

Lee YM, Ellis JH (1996) Comparison of algorithms for nonlinear integer optimization: application to monitoring network design. J Environ Eng-ASCE 122(6): 524-531

Massmann J, Freeze RA (1987) Groundwater contamination from waste management sites: the interaction between risk-based engineering design and regulatory policy, 1. Methodology. Water Resour Res 23(2): 351-367

McDonald MG, Harbaugh AW (1988) A modular three-dimensional finite-difference ground-water flow model. Book 6, United States Geological Survey, USA

Meyer PD, Valocchi AJ, Eheart JW (1994) Monitoring network design to provide initial detection of groundwater contamination. Water Resour Res 30(9): 2647-2659

Morris MD, Mitchell TJ (1995) Exploratory designs for computational experiments. J Stat Plan Infer 43: 381-402

Nunes LM, Cunha MC, Ribeiro L (2004a) Groundwater monitoring networks optimization with redundancy reduction. J Water Res PL - ASCE 130(1): 33-43

Nunes LM, Cunha MC, Ribeiro L (2004b) Groundwater nitrate monitoring network optimization with missing data. Water Resour Res 40(2): 1-18

Nunes LM, Cunha MC, Ribeiro L, Azevedo J (2005) New method for groundwater plume detection under uncertainty. In: Water in Celtic Countries: Quantity, Quality and Climate Variability, The Fourth Inter-Celtic Colloquium on Hydrology and Management of Water Resources, July 11-13, 2005, Guimarães, Portugal Olea RA (1999) Geostatistics for Engineers and Earth Scientists. Springer, New York 
294 Reed P, Minsker B, Valocchi AJ (2000) Cost-effective long-term groundwater 295 monitoring design using a genetic algorithm and global mass interpolation. Water Resour 296 Res 36(12): 3731-3741

297 Rouhani S (1985) Variance reduction analysis. Water Resour Res 21(6): 837-846

298 Royle JA, Nychka D (1998) An algorithm for the construction of spatial coverage designs 299 with implementation in SPLUS. Comput Geosci-UK 24(5): 479-488

300 Sorkin GB (1991) Efficient simulated annealing on fractal energy landscapes. 301 Algorithmica 6: 367-418

302 Zheng C (1990) MT3D: A modular three-dimensional transport model for simulation of 303 advection, dispersion and chemical reactions of contaminants in ground water systems. S. 304 S. Papadopulos and Assoc, USA.

305

306 
307

308

309

310

311

312

313

\section{FIGURES}

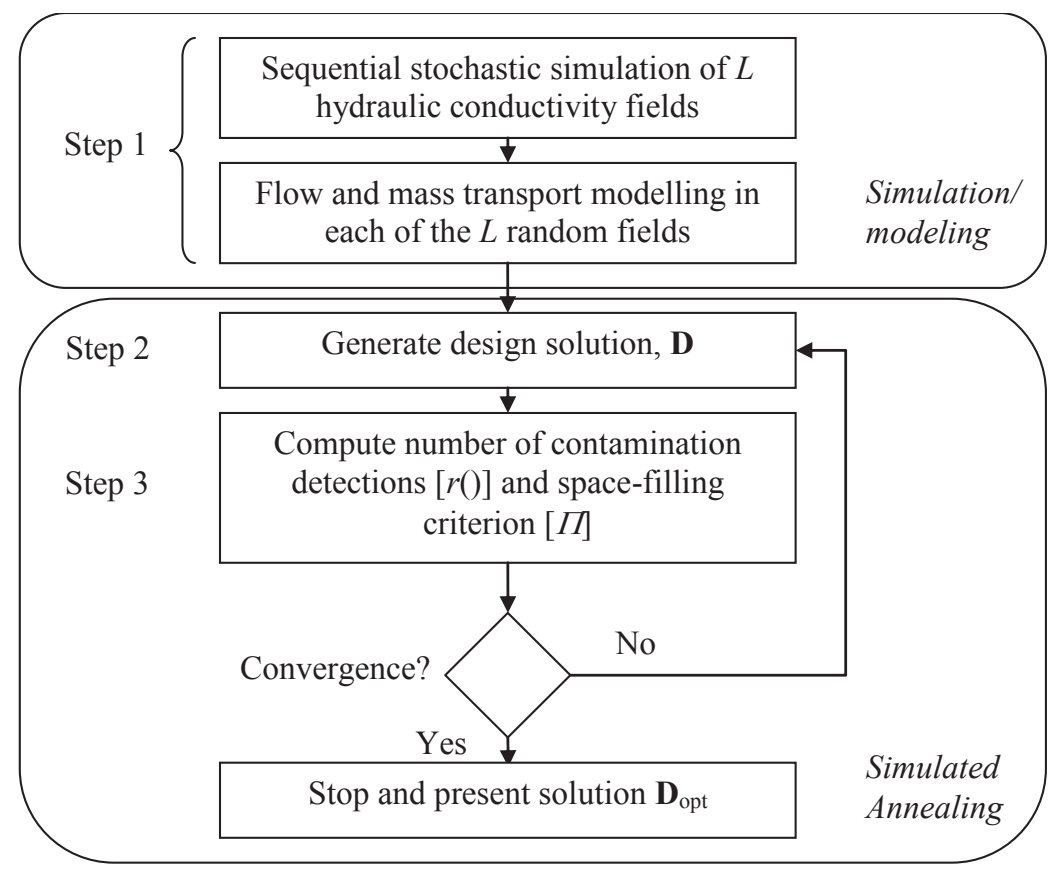

Figure 1. Method
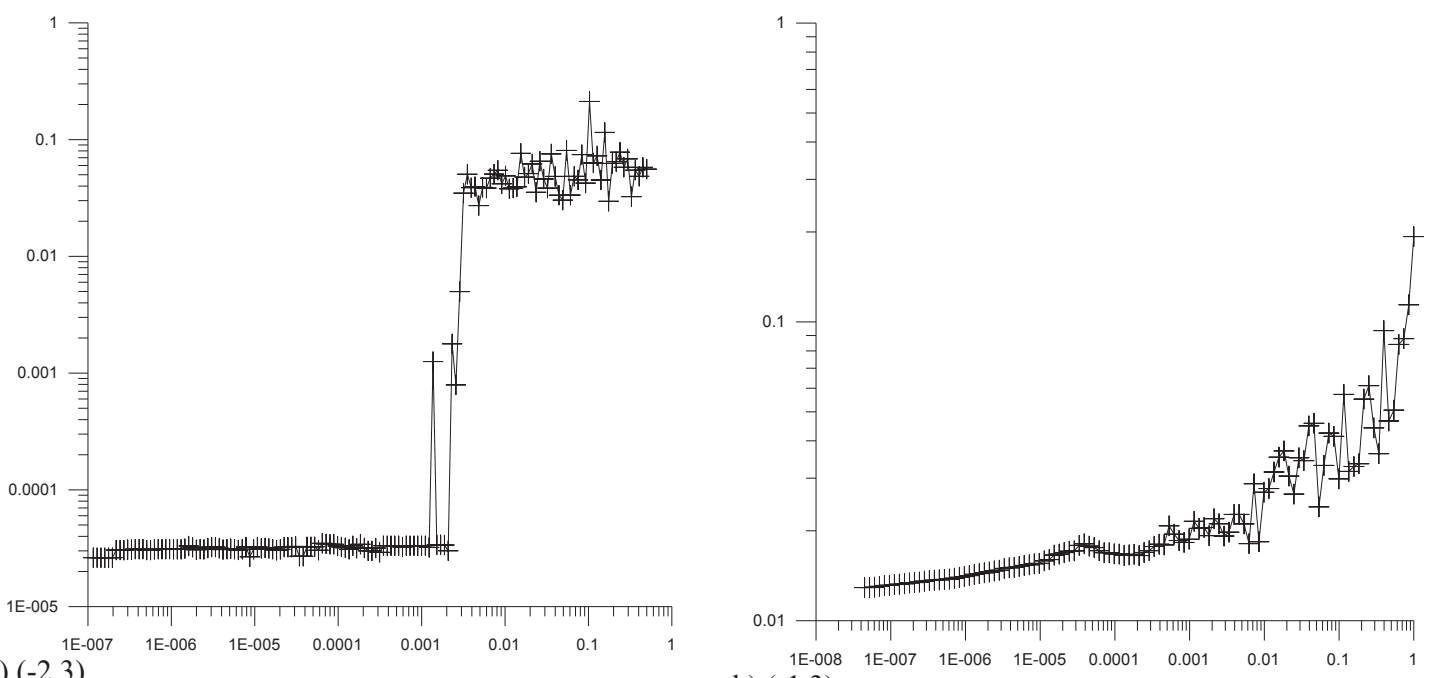

a) $(-2,3)$

b) $(-1,3)$

314 Figure 2. Simulated annealing convergence curves (best of the ten runs) 


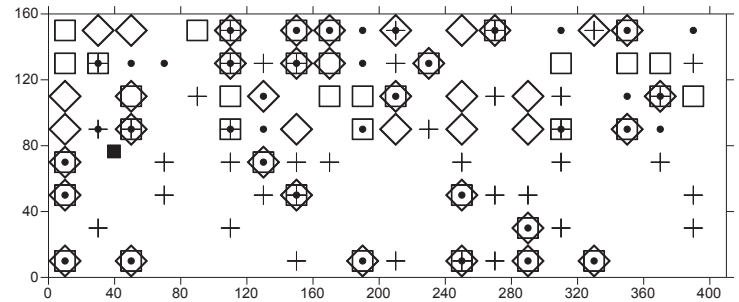

b) $(p, q)=+:(-2,3)$; $\square:(-1,3) ; \bullet:(-1,4) ; \diamond:(-3,4)$

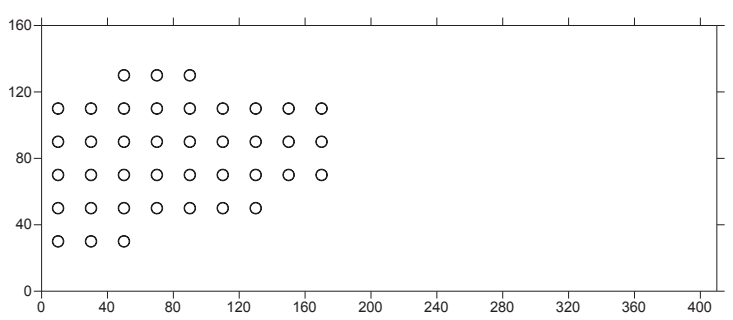

c)

317 Figure 3. a) Relative number of detections, $r_{C}\left(x_{\mathrm{i}, \mathrm{j}}\right)$; b) M1; c) M2

318

319

320 
Table 1. Comparison of results for objective functions M1 and M2 (best of the ten runs)

\begin{tabular}{lcccccc}
\hline \multicolumn{1}{c}{ Indicators } & $r_{C}\left(x_{\mathrm{i}, \mathrm{j}}\right)$ & $\mathrm{M} 2\left[r_{\boldsymbol{D}}\left(x_{\mathrm{i}, \mathrm{j}}\right)\right]$ & \multicolumn{4}{c}{$\mathrm{M} 1\left[r_{D}\left(x_{\mathrm{i}, \mathrm{j}}\right)\right]$} \\
\cline { 4 - 6 } & & & $p=-1 ; q=3$ & $p=-1 ; q=4$ & $p=-2 ; q=3$ & $p=-3 ; q=4$ \\
\hline Mean & 0.2970 & 0.24565 & 0.26572 & 0.26578 & 0.29538 & 0.26475 \\
Maximum & 0.7143 & 0.6780 & 0.7080 & 0.7080 & 0.7080 & 0.7080 \\
Minimum & 0.0 & 0.0 & 0.0 & 0.0 & 0.0 & 0.0 \\
Mean error & -0.00074 & -0.00205 & 0.00456 & 0.00668 & 0.00508 & 0.00425 \\
Relative mean error & - & 0.00830 & 0.0172 & 0.0251 & 0.0172 & 0.0161 \\
Mean Estimation Variance & 0.00240 & 0.0054 & 0.0053 & 0.0054 & 0.0056 & 0.0053 \\
Rank & - & 3 & 2 & - & 1 & - \\
\hline
\end{tabular}

